\title{
Actividades de evaluación continua - correlación con la calificación de la prueba final y efecto sobre la calificación final. Evidencia en Administración y Dirección de Empresas
}

\author{
Continuous assessment activities - correlation with \\ final exam grades and effect on final grades. A \\ business studies experience \\ Francisco JAREÑO CEBRIÁN y Raquel LÓPEZ GARCÍA \\ Universidad de Castilla-La Mancha
}

Recibido: Septiembre 2013

Aceptado: Noviembre 2013

\section{Resumen}

El presente artículo analiza las calificaciones parciales (de las distintas actividades de evaluación continua incluida la prueba final) y la calificación final (calculada como una media ponderada de las calificaciones parciales) para nueve asignaturas de tercer curso del Grado en Administración y Dirección de Empresas. Por un lado, los resultados muestran que existe una correlación positiva significativa entre las calificaciones del resto de actividades de evaluación continua y de la prueba final para siete asignaturas. Por otro lado, encontramos que, en general, la calificación obtenida en las distintas actividades de evaluación continua (excluida la prueba final) ayuda a mejorar la calificación final del estudiante. Es decir, la calificación final del estudiante es superior a la que obtendría si sólo se tuviera en cuenta el resultado de la prueba final. Los resultados son consistentes para un análisis por asignaturas y para una muestra formada por los estudiantes matriculados en las nueve asignaturas.

Palabras clave: educación superior, método de evaluación, evaluación del estudiante.

\begin{abstract}
In this paper we analyze the partial grades (of continuous assessment activities including the final exam) and the final grades (computed as a weighted average of partial grades) for nine subjects taught in the third year of Business Administration Degree. On one hand, results show that there is a significant positive correlation between the grades of continuous assessment activities (excluded the final exam) and final exam in seven subjects. On the other hand, overall we find that students' grades in continuous assessment activities (excluded the final exam) help in the improvement of their final grades (i.e., students' final grades exceed the grades that they would obtain by only taking into account their final exam grades). The results are consistent when the analysis is carried out over each subject area as well as over the grades of the students who registered for the nine subjects.
\end{abstract}

Keywords: higher education, evaluation methods, student evaluation. 
La Declaración de Bolonia de 19 de junio de 1999 sienta las bases para la construcción de un Espacio Europeo de Educación Superior (EEES) en un plazo de tiempo hasta 2010. El EEES implica un cambio en la estructura de los estudios de educación superior, que se organizan en dos ciclos principales (Grado y Posgrado), y en la manera de cuantificar y valorar el trabajo del estudiante en el proceso de aprendizaje, que se basa en el sistema ECTS (European Credit Transfer System o sistema europeo de transferencia y acumulación de créditos). El sistema ECTS supone realizar una evaluación continua de los conocimientos y de las competencias (capacidades, habilidades, destrezas y actitudes) adquiridas por el estudiante a través de las distintas actividades que se propongan, en lugar de una única evaluación basada en el examen o prueba final (sistema tradicional). Delgado y Oliver (2006) señalan algunas ventajas del sistema de evaluación continua tanto para el profesor como para el estudiante. Por un lado, al tratarse de una valoración integral, permite al profesor poder realizar un seguimiento más exhaustivo del progreso en el aprendizaje del estudiante. Por otro lado, la evaluación continua contribuye a aumentar la probabilidad de éxito de los estudiantes por factores tales como la asimilación y desarrollo gradual de conocimientos y competencias, y la información cualitativa (retroalimentación) que reciben los estudiantes sobre los puntos fuertes y débiles de su trabajo para mejorar su proceso de aprendizaje (evaluación formativa).

A raíz del reciente proceso de adaptación de las Universidades españolas al nuevo sistema de enseñanza-aprendizaje, podemos encontrar diversos estudios que analizan los resultados del sistema de evaluación continua y el grado de satisfacción de los estudiantes en distintas disciplinas de estudio (véase Jareño, 2007; López et al., 2007; Mingorance, 2008; Amo et al., 2014; Carrillo-de-la-Peña et al., 2009; Gracia y Pinar, 2009; Herradón et al., 2009; Florido et al., 2011; Carrillo-de-la-Peña y Pérez, 2012; Ricoy y Fernández-Rodríguez, 2013). De aquellos estudios que comparan los resultados del sistema de evaluación continua frente al sistema de evaluación tradicional se desprenden los siguientes resultados: aumenta el porcentaje de aprobados (Mingorance, 2008; Gracia y Pinar, 2009; Carrillo-de-la-Peña y Pérez, 2012); aumenta la calificación final (Jareño, 2007; López et al., 2007; Carrillo-de-la-Peña et al., 2009; Gracia y Pinar, 2009; Carrillo-de-la-Peña y Pérez, 2012); y se reduce la dispersión en las calificaciones (es decir, se reduce el número de calificaciones bajas y el número de calificaciones altas) (López et al., 2007; Mingorance, 2008).

Los resultados de los anteriores estudios son los obtenidos para dos muestras de estudiantes de una determinada asignatura durante un curso académico concreto (estudiantes seguidores del sistema de evaluación continua frente a estudiantes seguidores del sistema tradicional), o bien para dos muestras de estudiantes de una determinada asignatura en distintos cursos académicos (antes y después de la implantación del sistema ECTS). Es decir, los estudiantes de las muestras cuyas calificaciones finales se comparan son distintos. En todos estos trabajos, la calificación final en el sistema de evaluación continua se obtiene como una media ponderada de las calificaciones obtenidas por los estudiantes en las distintas actividades de evaluación continua que, de acuerdo con la guía docente de la asignatura, tienen un reconocimiento en la calificación final. A lo largo de este trabajo utilizaremos el 
concepto de evaluación continua calificativa para referirnos a la calificación final obtenida de esta forma.

El presente estudio difiere de los anteriormente mencionados en dos aspectos. Por un lado, contamos con una única muestra formada por los estudiantes de nueve asignaturas de tercer curso del Grado en Administración y Dirección de Empresas (ADE) en las que se sigue una evaluación continua calificativa. Por otro lado, no utilizamos sólo las calificaciones finales, sino que analizamos la relación entre tres calificaciones: la calificación de la prueba final, la calificación del resto de actividades de evaluación continua y la calificación final. Es decir, la calificación final en todas las asignaturas se obtiene como una media ponderada de la calificación de las distintas actividades de evaluación continua (incluida la prueba final).

En concreto, a partir de los datos recogidos se pretenden analizar dos cuestiones. En primer lugar, estudiamos la correlación entre las calificaciones del resto de actividades de evaluación continua y de la prueba final para cada asignatura. Se trata de investigar si, además de la función de información al estudiante sobre su proceso de aprendizaje que cumple la evaluación continua, superar con éxito las actividades propuestas durante el curso se relaciona positivamente con la calificación obtenida en la prueba final. En segundo lugar, analizamos si existen diferencias significativas entre la calificación final que efectivamente obtienen los estudiantes y la que obtendrían si sólo se tuviera en cuenta el resultado de la prueba final. Es decir, investigamos si la evaluación continua calificativa tiene un efecto positivo o negativo sobre la calificación final del estudiante. Esta última cuestión se analiza primero a partir de las calificaciones para cada asignatura, y después para una muestra formada por los estudiantes matriculados en las nueve asignaturas. El análisis por asignaturas permite cubrir un espectro más amplio de estudiantes al incluir también estudiantes que no tienen por qué estar matriculados en las nueve asignaturas de tercer curso. Los resultados obtenidos si sólo se utilizara la muestra común podrían estar sesgados porque los estudiantes matriculados en las nueve asignaturas de tercer curso es probable que respondan a un perfil concreto de estudiantes que han ido superando las asignaturas de cada curso y, por lo tanto, se identifican con los mejores estudiantes.

\section{Metodología}

Para dar respuesta a las dos cuestiones planteadas se cuenta con una muestra formada por los estudiantes de tercer curso del Grado en ADE de la Facultad de Ciencias Económicas y Empresariales de Albacete (Universidad de Castilla-La Mancha) del curso académico 2011-2012. El Grado en ADE comenzó a impartirse por primera vez en el curso académico 2009-2010. Por lo tanto, los resultados de este trabajo corresponden a la primera promoción del Grado en ADE en el último curso en el que se imparten asignaturas obligatorias. ${ }^{1}$ La docencia en el tercer curso se imparte

\footnotetext{
${ }^{1}$ En el cuarto curso los estudiantes cursan asignaturas optativas (primer semestre) y realizan prácticas en empresas y el trabajo fin de grado (segundo semestre).
} 
en dos grupos - grupo de mañana y grupo de tarde - de manera que los estudiantes de ambos grupos forman parte de la muestra.

En cuanto a las variables analizadas, se trata de la calificación de la prueba final (PF), la calificación obtenida en el resto de actividades de evaluación continua (EC) y la calificación final $(\mathrm{CF})$ en nueve asignaturas. La variable $\mathrm{CF}$ se calcula como:

$$
C F=\sum_{i=1}^{n} w_{E C_{i}} E C_{i}+w_{P F} P F \text {, siendo } \sum_{i=1}^{n} w_{E C_{i}}+w_{P F}=1,
$$

donde $w_{E C i}$ es el peso que tiene la actividad de evaluación continua $i$-ésima, $E C_{i}$, sobre CF y $w_{P F}$ es el peso que tiene PF sobre CF, siendo $n$ el número de actividades de evaluación continua además de la prueba final. ${ }^{2}$ Para cada asignatura se incluyen sólo las calificaciones mayores que cero en la prueba final y en al menos una del resto de actividades de evaluación continua en la convocatoria ordinaria. ${ }^{3}$ El motivo de que sólo consideremos los resultados de la convocatoria ordinaria es que creemos que la correlación entre el resto de actividades de evaluación continua y la prueba final es mayor cuanto menor es el tiempo que transcurre entre la realización de los mismos.

El nombre de las asignaturas, el peso de las distintas actividades de evaluación continua sobre $\mathrm{CF}$ y el número de observaciones en cada asignatura aparecen recogidos en la Tabla I del Anexo 1. Observamos que en siete asignaturas, PF supone el $60 \%$ o el $70 \%$ de $\mathrm{CF}$; en una asignatura, EC y $\mathrm{PF}$ tienen el mismo peso; y en la asignatura restante, el peso de $\mathrm{PF}$ sobre $\mathrm{CF}$ es menor que el de EC.

El análisis estadístico de las variables "calificación" es el siguiente. La correlación entre las variables EC y PF se analiza para cada asignatura utilizando el coeficiente de correlación lineal de Pearson y midiendo su significación estadística a partir de la prueba $t$ de Student. Para investigar las diferencias entre las variables CF y PF se calcula la variable DIF para todos los estudiantes de la muestra:

$$
\mathrm{DIF}=\mathrm{CF}-\mathrm{PF}^{4}
$$

DIF se analiza, primero, para cada asignatura y, después, para una muestra formada por 25 estudiantes matriculados en las nueve asignaturas.

En el análisis por asignaturas de DIF, se presentan en primer lugar los principales estadísticos descriptivos (media, mediana y desviación típica). Entonces se utilizan la prueba $t$ de Student y la prueba de rangos con signo de Wilcoxon para determinar si la

\footnotetext{
${ }^{2}$ Es decir, utilizamos la CF "objetiva”, no la que aparece en el expediente del estudiante. Entendemos que cualquier (leve) diferencia entre una y otra responde al criterio subjetivo del profesor.

${ }^{3}$ Además, utilizamos la guía docente de cada asignatura para saber si el cómputo de la ponderación de alguna actividad está sujeto a alguna restricción, como puede ser el haber alcanzado una nota mínima.

4 Amo y Jareño (2011) proponen una medida "DIF" para analizar la distancia entre la evaluación que los estudiantes conceden al trabajo realizado en una presentación en grupo (inter e intra grupos) y la otorgada por los profesores. Así, se estudia la adquisición de competencias tales como la responsabilidad, el juicio crítico, autoaprendizaje..., ya que se comparan resultados de dos niveles diferentes (primero y tercero de la titulación en "Administración y Dirección de Empresas"). Jareño et al. (2013) analizan otra medida "DIF" para estudiar la diferente percepción que los estudiantes (a nivel individual) tienen acerca de su contribución al trabajo cooperativo, en relación a la percibida por los compañeros de grupo.
} 
media y la mediana de DIF, respectivamente, son significativamente distintas de cero. ${ }^{5}$ Además se utiliza un gráfico de barras para visualizar los resultados (signo y magnitud de la media de DIF para cada asignatura). A continuación, se presenta la distribución en porcentaje de DIF atendiendo a su signo $(\mathrm{DIF}>0, \mathrm{DIF}=0$ y $\mathrm{DIF}<0)$ mediante gráficos circulares. Por último, utilizamos gráficos de barras para mostrar el porcentaje de estudiantes que, habiendo suspendido la prueba final, han aprobado la asignatura como resultado de la evaluación continua calificativa:

$$
\left(\mathrm{N}^{o} \text { estudiantes con } \mathrm{PF}<5\right) /\left(\mathrm{N}^{o} \text { estudiantes con } \mathrm{CF}>4.9\right) \text {, }
$$

así como el porcentaje de estudiantes que aun superando la prueba final, tienen un suspenso en la CF:

$$
\text { ( } \left.\mathrm{N}^{\mathrm{o}} \text { estudiantes con } \mathrm{PF} \geq 5\right) /\left(\mathrm{N}^{\mathrm{o}} \text { estudiantes con } \mathrm{CF} \leq 4.9\right){ }^{6}
$$

Finalmente, en el análisis de la muestra común se exhibe el número de estudiantes para los que $\mathrm{DIF}>0, \mathrm{DIF}=0$ y $\mathrm{DIF}<0$ en ninguna, una, ..., nueve de las asignaturas en las que están matriculados.

\section{Resultados}

En este apartado primero se analiza la correlación entre EC y PF para cada asignatura. Después se analizan las diferencias entre PF y CF para cada asignatura y para una muestra de 25 estudiantes matriculados en las nueve asignaturas.

\section{Correlación entre EC y PF}

Existe consistente evidencia empírica en la literatura sobre el efecto positivo que la evaluación continua tiene en el éxito académico de los estudiantes (véase Mingorance, 2008; Carrillo-de-la-Peña et al., 2009; Gracia y Pinar, 2009; Peterson y Siadat, 2009; Carrillo-de-la-Peña y Pérez, 2012, entre otros). En concreto, Carrillo-de-la-Peña et al. (2009) y Peterson y Siadat (2009) muestran que la realización de actividades de evaluación continua (independientemente del éxito obtenido) se relaciona positivamente con $\mathrm{CF}$ y $\mathrm{PF}$, respectivamente. En este trabajo analizamos la relación que existe entre el éxito obtenido en la realización del resto de actividades de evaluación continua y el resultado de la prueba final. Es decir, estudiamos la correlación entre EC y PF.

La Tabla II muestra el coeficiente de correlación lineal de Pearson, $r$, y su significación estadística para las nueve asignaturas. Dicho coeficiente puede tomar valores comprendidos entre -1 y 1 , donde -1 (1) significa que existe una relación lineal negativa (positiva) perfecta entre las variables, mientras que un coeficiente próximo a cero quiere decir que no existe relación lineal entre las variables.

\footnotetext{
${ }^{5}$ Utilizamos la prueba no paramétrica de rangos con signo de Wilcoxon además de la prueba $t$ de Student porque esta última se aplica cuando la serie sigue una distribución normal, y de acuerdo con la prueba de normalidad de Jarque-Bera la variable DIF no sigue una distribución normal para algunas asignaturas.

${ }^{6}$ Consideramos que $\mathrm{CF}>4.9$ es aprobado.
} 


\begin{tabular}{lccccc}
\hline & AC & CC & DCE & DF & DO \\
\hline$r$ & 0.34 & 0.39 & 0.06 & 0.38 & 0.17 \\
\hline $\begin{array}{l}\text { Prueba } t \text { de Student } \\
\left(\mathrm{H}_{0}: r=0\right)\end{array}$ & $2.96^{\mathrm{c}}$ & $3.77^{\mathrm{c}}$ & 0.66 & $3.60^{\mathrm{c}}$ & 1.65 \\
\hline$r$ & $\mathbf{E E}$ & FE & IEIE & IM & \\
\hline $\begin{array}{l}\text { Prueba } t \text { de Student } \\
\left(\mathrm{H}_{0}: r=0\right)\end{array}$ & 0.45 & 0.26 & 0.22 & 0.23 & \\
\hline
\end{tabular}

Tabla II. Coeficiente de correlación lineal de Pearson, $r$, entre EC y PF. La hipótesis nula de la prueba $t$ de Student es que el coeficiente de correlación es igual a cero. Los $p$-valores son: ${ }^{a} \mathrm{p}<$ $0.10,{ }^{\mathrm{b}} \mathrm{p}<0.05,{ }^{\mathrm{c}} \mathrm{p}<0.01$.

Observamos que existe una correlación positiva estadísticamente significativa entre EC y PF para siete de las nueve asignaturas. En cuatro asignaturas, los coeficientes de correlación son superiores al $30 \%$, todos ellos significativos al 1\%. El máximo coeficiente de correlación es del 45\%. La evidencia encontrada de una correlación positiva significativa entre EC y PF para más de la mitad de las asignaturas apoya la hipótesis de que el éxito de los estudiantes en la prueba final se relaciona positivamente no sólo con la realización de actividades de evaluación continua, sino también con el éxito obtenido en la resolución de las mismas. Así, el éxito en la resolución de las actividades de evaluación continua revela que el estudiante ha asimilado correctamente los conocimientos y esto se traduce en el resultado de la prueba final.

\section{Diferencias entre CF y PF}

En este apartado se analiza la variable DIF. Primero de manera separada para cada una de las nueve asignaturas, y después para una muestra formada por 25 estudiantes matriculados en las nueve asignaturas.

\section{a) Análisis por asignaturas}

La Tabla III recoge la media, la mediana y la desviación típica de DIF para cada asignatura junto con la significación estadística de la media y la mediana. 


\begin{tabular}{|c|c|c|c|c|c|}
\hline & AC & $\mathbf{C C}$ & DCE & $\overline{\text { DF }}$ & DO \\
\hline Media $(\mu)$ & 0.76 & 1.11 & 0.20 & -0.51 & 0.35 \\
\hline $\begin{array}{l}\text { Prueba } t \text { de Student } \\
\left(\mathrm{H}_{0}: \mu=0\right)\end{array}$ & $14.01^{\mathrm{c}}$ & $10.72^{\mathrm{c}}$ & $3.18^{\mathrm{c}}$ & $-6.50^{c}$ & $4.77^{\mathrm{c}}$ \\
\hline Mediana $(M)$ & 0.72 & 0.78 & 0.20 & -0.61 & 0.37 \\
\hline $\begin{array}{l}\text { Prueba de rangos con } \\
\text { signo de Wilcoxon } \\
\left(\mathrm{H}_{0}: M=0\right)\end{array}$ & $7.15^{\mathrm{c}}$ & $7.54^{\mathrm{c}}$ & $3.14^{\mathrm{c}}$ & $5.30^{\mathrm{c}}$ & $4.51^{\mathrm{c}}$ \\
\hline \multirow[t]{2}{*}{ Desv. típica } & 0.45 & 0.92 & 0.64 & 0.68 & 0.71 \\
\hline & $\mathbf{E E}$ & FE & IEIE & IM & \\
\hline Media $(\mu)$ & 0.31 & 0.04 & 0.23 & 1.60 & \\
\hline $\begin{array}{l}\text { Prueba } t \text { de Student } \\
\left(\mathrm{H}_{0}: \mu=0\right)\end{array}$ & $3.12^{\mathrm{c}}$ & 0.25 & $4.10^{\mathrm{c}}$ & $17.57^{\mathrm{c}}$ & \\
\hline Mediana $(M)$ & 0.33 & -0.06 & 0.00 & 1.70 & \\
\hline $\begin{array}{l}\text { Prueba de rangos con } \\
\text { signo de Wilcoxon } \\
\left(\mathrm{H}_{0}: M=0\right)\end{array}$ & $3.19^{\mathrm{c}}$ & 0.01 & $3.81^{\mathrm{c}}$ & $8.02^{\mathrm{c}}$ & \\
\hline Desv. típica & 0.86 & 1.28 & 0.43 & 0.85 & \\
\hline
\end{tabular}

Tabla III. Media, mediana y desviación típica de DIF. La hipótesis nula de la prueba $t$ de Student y de la prueba de rangos con signo de Wilcoxon es que la media y la mediana son iguales a cero, respectivamente. Los $p$-valores son: ${ }^{\mathrm{a}} \mathrm{p}<0.10,{ }^{\mathrm{b}} \mathrm{p}<0.05,{ }^{\mathrm{c}} \mathrm{p}<0.01$.

De acuerdo con los resultados obtenidos, la media de DIF es significativamente distinta de cero en ocho asignaturas. Es decir, en ocho de las nueve asignaturas existen, de media, diferencias estadísticamente significativas entre CF y PF. Lo mismo es cierto para la mediana. En cuanto al signo de la media de DIF, observamos que es positivo para siete de las ocho asignaturas en las que ésta es significativamente distinta de cero. Dicho de otro modo, CF es de media mayor (menor) que PF en siete (una) asignaturas. Para una rápida visualización de la magnitud y el signo de la media de DIF, se presentan los resultados en un gráfico de barras (Gráfico I). 


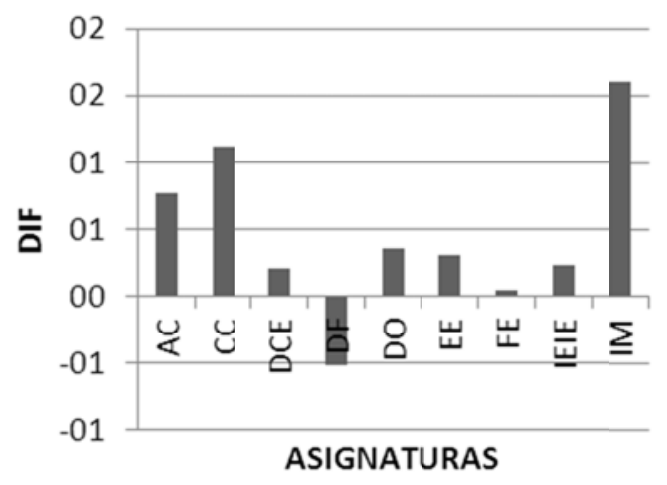

Gráfico I. Media de la variable DIF.

A continuación, analizamos la distribución en porcentaje de DIF en función de su signo $(\mathrm{DIF}>0, \mathrm{DIF}=0$ y $\mathrm{DIF}<0$ ). Los resultados aparecen recogidos en el Gráfico II. Observamos que en seis asignaturas el porcentaje de DIF $>0$ es superior al $50 \%$ - para tres asignaturas este porcentaje está por encima del $90 \%$. En cuanto a $\mathrm{DIF}<0$, para más de la mitad de los estudiantes $\mathrm{DIF}<0$ en dos asignaturas. Por último, en una asignatura $\mathrm{DIF}=0$ para el $58 \%$ de los estudiantes.

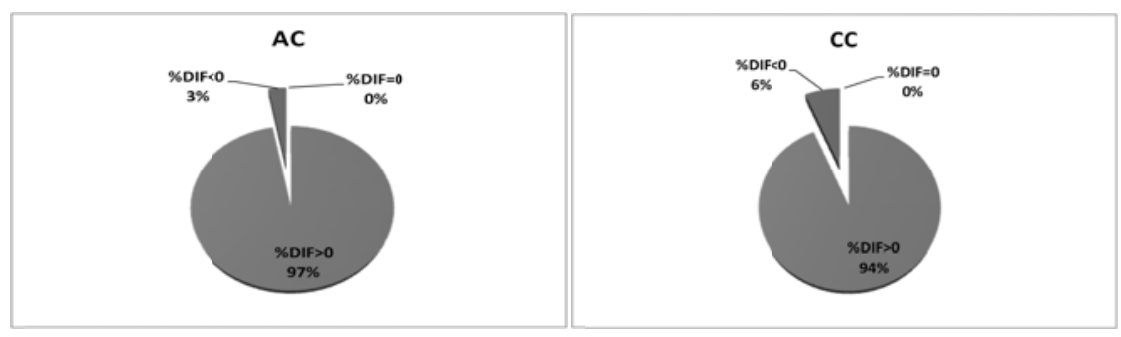

DCE



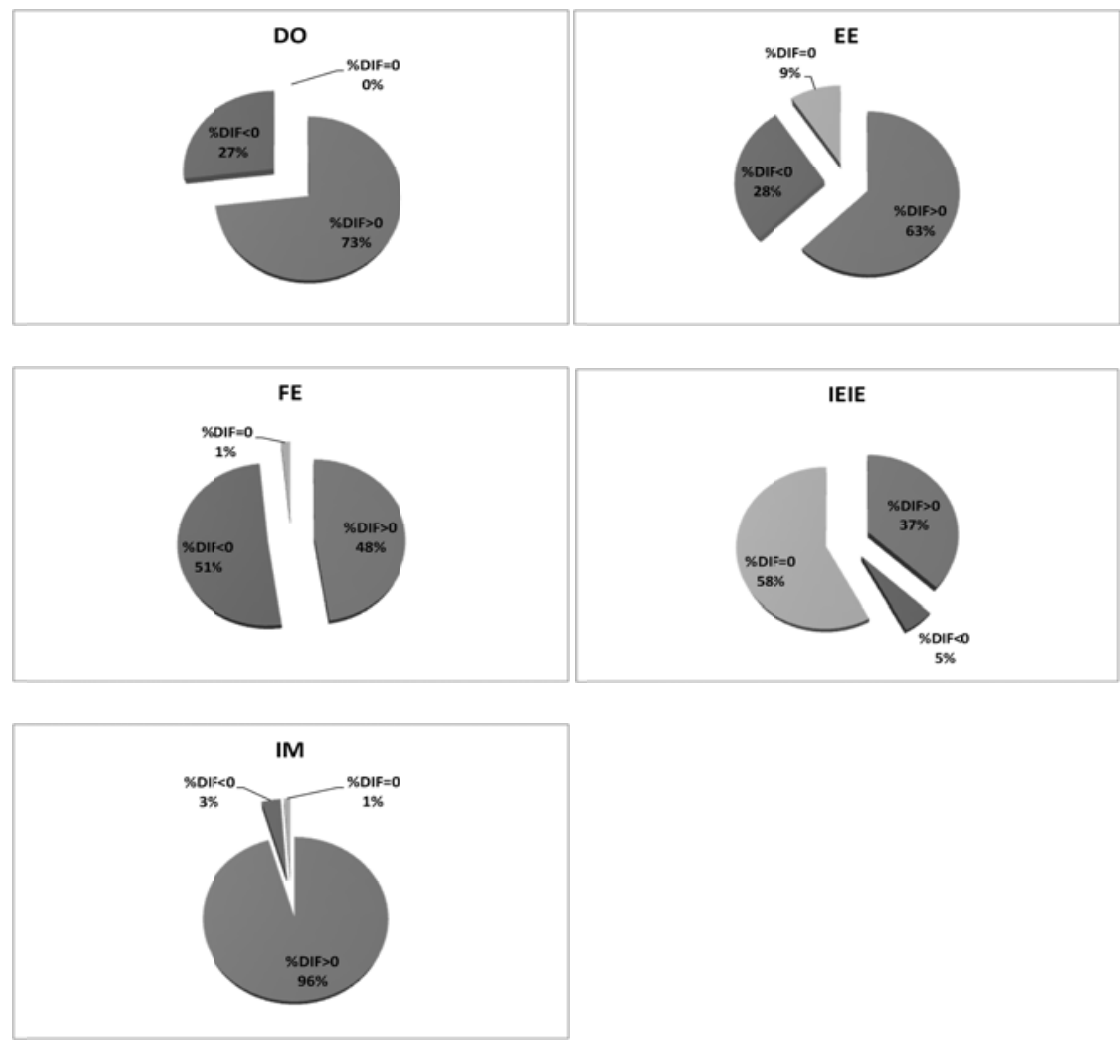

Gráfico II. Porcentajes de distribución de DIF en DIF $>0$, DIF=0 y DIF $<0$.

En definitiva, los resultados obtenidos hasta el momento evidencian que, en términos generales, la evaluación continua calificativa ayuda a mejorar la CF de los estudiantes, es decir, su CF es superior a la que obtendrían si sólo se tuviera en cuenta PF.

Por último, vamos un paso más allá en nuestro análisis, y calculamos el porcentaje de aprobados y de suspensos como resultado de la evaluación continua calificativa, es decir, el porcentaje de estudiantes que habiendo suspendido (aprobado) la prueba final, aprueban (suspenden) al calcular CF. Los resultados aparecen recogidos en los Gráficos III y IV, respectivamente. 


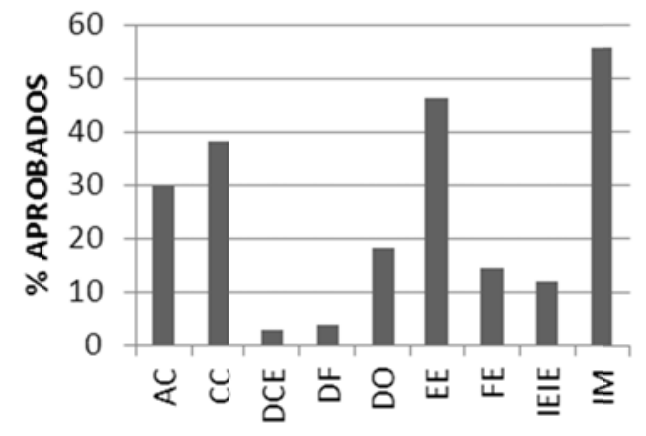

ASIGNATURAS

Gráfico III. Porcentaje de aprobados como resultado de la evaluación continua calificativa.

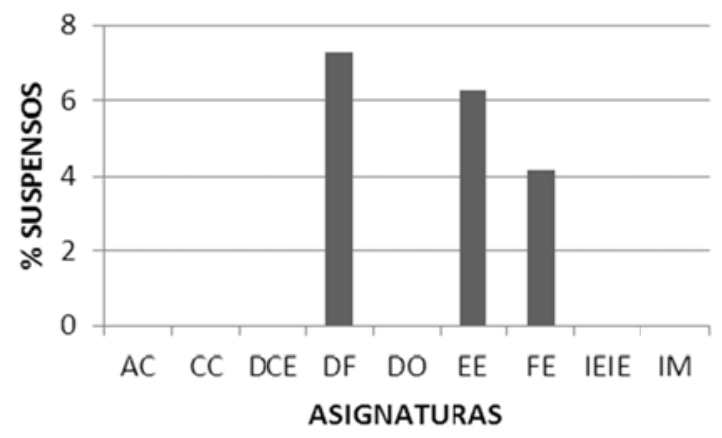

Gráfico IV. Porcentaje de suspensos como resultado de la evaluación continua calificativa.

De acuerdo con el Gráfico III, en cuatro asignaturas más del 25\% de los estudiantes aprobados lo han hecho gracias al reconocimiento que ha tenido $\mathrm{EC}$ en $\mathrm{CF}$. Este porcentaje es superior al $50 \%$ en una asignatura. Comprobamos que sólo en dos asignaturas el porcentaje de aprobados como resultado de la evaluación continua calificativa es inferior al 5\%. Por su parte, el gráfico IV revela que sólo en tres asignaturas existen estudiantes que suspenden al incluir $\mathrm{EC}$ en $\mathrm{CF}$, y que el porcentaje de suspensos es inferior al $8 \%$ en todos los casos.

De esta manera, podemos concluir que, en términos generales, el porcentaje de aprobados como resultado de la evaluación continua calificativa supera notablemente al de suspensos - sólo hay una asignatura en la que el porcentaje de estudiantes que suspenden (7\% aprox.) supera al de aprobados (4\% aprox.). 


\section{b) Análisis de la muestra común}

Para una muestra formada por 25 estudiantes matriculados en las nueve asignaturas, la Tabla IV recoge el número de estudiantes para los que $\mathrm{DIF}>0$, DIF $=0$, y $\mathrm{DIF}<0$ en ninguna, una,..., nueve asignaturas.

\begin{tabular}{lcccccccccc}
\hline \multicolumn{1}{c}{ Número de asignaturas } \\
\hline & $\mathbf{0}$ & $\mathbf{1}$ & $\mathbf{2}$ & $\mathbf{3}$ & $\mathbf{4}$ & $\mathbf{5}$ & $\mathbf{6}$ & $\mathbf{7}$ & $\mathbf{8}$ & $\mathbf{9}$ \\
\hline Estudiantes con DIF $>0$ & 0 & 0 & 0 & 0 & 0 & 5 & 8 & 8 & 4 & 0 \\
\hline Estudiantes con DIF $=0$ & 14 & 10 & 1 & 0 & 0 & 0 & 0 & 0 & 0 & 0 \\
\hline Estudiantes con DIF $<0$ & 2 & 5 & 10 & 5 & 3 & 0 & 0 & 0 & 0 & 0 \\
\hline
\end{tabular}

Tabla IV. Número de estudiantes para los que DIF $>0$, DIF $=0$ y $\mathrm{DIF}<0$ en ninguna, una,...,

nueve asignaturas.

Los resultados obtenidos muestran que DIF $>0$ para todos los estudiantes en al menos cinco asignaturas. El resultado más favorable se presenta para cuatro estudiantes para los cuales DIF $>0$ en ocho de las nueve asignaturas. Encontramos también que sólo hay estudiantes para los que $\mathrm{DIF}=0$ en una o dos asignaturas. En cuanto a $\mathrm{DIF}<0$, hay tres estudiantes para los que $\mathrm{DIF}<0$ en cuatro asignaturas, siendo éste el resultado más desfavorable. Es decir, para el resto de estudiantes DIF $<0$ en ninguna, una, dos o tres asignaturas.

En general, vemos cómo la concentración de estudiantes en un número más elevado de asignaturas ocurre para la variable DIF $>0$. Es decir, el número de asignaturas en las que los estudiantes obtienen $\mathrm{CF}>\mathrm{PF}$ es mayor que el número de asignaturas en las que $\mathrm{CF}=\mathrm{PF}$ o $\mathrm{CF}<\mathrm{PF}$. Por lo tanto, los resultados obtenidos para la muestra común de estudiantes son consistentes con los obtenidos para las muestras individuales por asignaturas, y que evidencian el efecto positivo de la evaluación continua calificativa sobre el resultado académico de los estudiantes de tercer curso del Grado en ADE.

\section{Conclusiones}

En este trabajo analizamos la relación que existe entre tres calificaciones - la calificación de la prueba final (PF), la calificación del resto de actividades de evaluación continua (EC) y la calificación final (CF) - para 700 estudiantes de nueve asignaturas de tercer curso del Grado en ADE. En todas las asignaturas, CF se obtiene como una media ponderada de las calificaciones de las distintas actividades de evaluación continua (incluida PF).

Por un lado, encontramos que existe una correlación positiva estadísticamente significativa entre EC y PF para siete asignaturas. Es decir, el éxito en la prueba final se relaciona positivamente con el éxito obtenido en la resolución del resto de actividades de evaluación continua. Los coeficientes de correlación lineal van desde el $22 \%$ al $45 \%$.

Por otro lado, los resultados obtenidos al comparar CF y PF a través de la variable DIF $(\mathrm{DIF}=\mathrm{CF}-\mathrm{PF}$ ) revelan que la media de DIF es significativamente distinta de 
cero en ocho asignaturas. En cuanto al signo de DIF, encontramos que CF es de media superior a PF en siete asignaturas, y que en seis (dos) asignaturas CF es mayor (menor) que PF para más de la mitad de los estudiantes. Además, en siete asignaturas existe un porcentaje superior al $10 \%$ de estudiantes que tienen aprobado en $\mathrm{CF}$ habiendo suspendido la prueba final. Sólo en una asignatura el anterior porcentaje es inferior al porcentaje de estudiantes que suspenden habiendo aprobado la prueba final. Por lo tanto, los resultados de este trabajo revelan que, en general, CF que efectivamente obtienen los estudiantes es superior a la que obtendrían si sólo se tuviera en cuenta PF. Es decir, EC ayuda a mejorar el resultado académico de los estudiantes. Los resultados son consistentes cuando DIF se analiza para una submuestra formada por 25 estudiantes matriculados en las nueve asignaturas.

\section{Referencias bibliográficas}

AMO, E. Y JAREÑO, F. (2011). Self, peer and teacher assessment as active learning methods. Research Journal of International Studies, 18, 41-47.

AMO, E.; JAREÑO, F.; LAGOS, M.G. Y TOBARRA, M.A. (2014). Las nuevas metodologías docentes y su repercusión en los planes de estudio. Innovar: Revista de Ciencias Administrativas y Sociales, 24(54), 231-249.

CARRILLO-DE-LA-PEÑA, M.T.; BAILLÈS, E.; CASERAS, X.; MARTÍNEZ, A.; ORTET, G. Y PÉREZ, J. (2009). Formative assessment and academic achievement in pre-graduate students of health sciences. Advances in health sciences education: theory and practice, 14(1), 61-67.

CARRILLO-DE-LA-PEÑA, M.T. Y PÉREZ, J. (2012). Continuous assessment improved academic achievement and satisfaction of psychology students in Spain. Teaching of Psychology, 39 (1), 45-47.

DELGADO, A.M. Y OLIVER, R. (2006). La evaluación continua en un nuevo escenario docente. Revista de Universidad y Sociedad del Conocimiento (RUSC), 3 (1), 1-12.

ESCRIBANO, F. Y JAREÑO, F. (2013). Aplicación de la metodología ABP en el Máster Universitario en Consultoría y Asesoría Financiera y Fiscal, en E. Farinós, y A. Escribano (ed.), Experiencias de Innovación Educativa en Finanzas, 73-87. Valencia: Tirant Lo Blanch.

FLORIDO, C.; JIMÉNEZ, J.L. Y SANTANA, I. (2011). Obstáculos en el camino hacia Bolonia: efectos de la implantación del Espacio Europeo de la Educación Superior (EEES) sobre los resultados académicos. Revista de Educación, 354, 629656.

GRACIA, J. Y PINAR, M.A. (2009). Una experiencia práctica de evaluación por competencias mediante el uso del portafolio del estudiante y su impacto temporal. Revista de Formación e Innovación Educativa Universitaria, 2 (2), 76-86. 
HERRADÓN, R., BLANCO, J., PÉREZ, A. Y SÁNCHEZ, J.A. (2009). Experiencias y metodologías "b-learning" para la formación y evaluación en competencias genéricas en Ingeniería. La Cuestión Universitaria, 5, 33-45.

JAREÑO, F. (2007). Espacio Europeo de Educación Superior: una propuesta de trabajo en Economía Financiera sobre la base de la experiencia previa, en M. R. Pardo; E. Amo; C. Córcoles; A. Tejada, y J. García (ed.), I Evaluación de la Implantación de Cursos Piloto en Economía y Administración de Empresas, 136-146. Albacete: Facultad CC Económicas y Empresariales de Albacete.

JAREÑO, F. (2011). Experiencia docente en la Facultad de CC. Económicas y Empresariales de Albacete, en E. Farinós y P. Soriano (ed.), Innovación Educativa en Finanzas en la implantación del Espacio Europeo de Educación Superior, 1-20. Valencia: Reproexpres Ediciones.

JAREÑO, F.; JIMÉNEZ, J.J. Y LAGOS, M.G. (2013). Aprendizaje cooperativo en educación superior: diferencias en la percepción de la contribución al grupo, en C. Díaz e I. Novo (ed.), V Jornadas de Docencia en Economía, 44. Toledo: Copicentro S.L.

JIMÉNEZ, J.J., LAGOS, M.G. Y JAREÑO, F. (2011). Una experiencia interdisciplinar de Aprendizaje Basado en Problemas con estudiantes de Administración y Dirección de Empresas, en M. Marín; A. B. Morales; I. y D. Delgado (ed.), Trabajos Fin de Grado y Máster: La Evaluación Global, 81-88. Toledo: Unidad de Innovación Educativa UCLM.

LÓPEZ, D.; HERRERO, J.R.; PAJUELO, A. Y DURÁN, A. (2007). A Proposal for Continuous Assessment at Low Cost. $37^{\text {th }}$ ASEE/IEEE Frontiers in Education Conference.

MINGORANCE, A.C. (2008). Análisis comparado entre los resultados de una evaluación continua y otra puntual. El caso de la asignatura macroeconomía. Revista de Investigación Educativa, 26 (1), 95-120.

PETERSON, E. Y SIADAT, M.V. (2009). Combination of formative and summative assessment instruments in elementary algebra classes: A prescription for success. Journal of Applied Research in the Community College, 16(2), 92-102.

RICOY, M.C. Y FERNÁNDEZ-RODRÍGUEZ, J. (2013). La percepción que tienen los estudiantes universitarios sobre la evaluación: Un estudio de caso. Educación XX1, $16(2), 321-342$.

\section{Correspondencia con los autores}

Raquel LÓPEZ GARCÍA

Facultad de Ciencias Económicas y Empresariales

Plaza de la Universidad 1, 02071, Albacete

e-mail: raquel.lopez@uclm.es

Teléfono: 967- 599200; Ext: 2356 


\section{Francisco JAREÑO CEBRIÁN}

Facultad de Ciencias Económicas y Empresariales,

Universidad de Castilla-La Mancha,

Plaza de la Universidad 1, 02071, Albacete

e-mail: francisco.jareno@uclm.es

Teléfono: 967- 599200; Ext: 2376

ANEXO 1

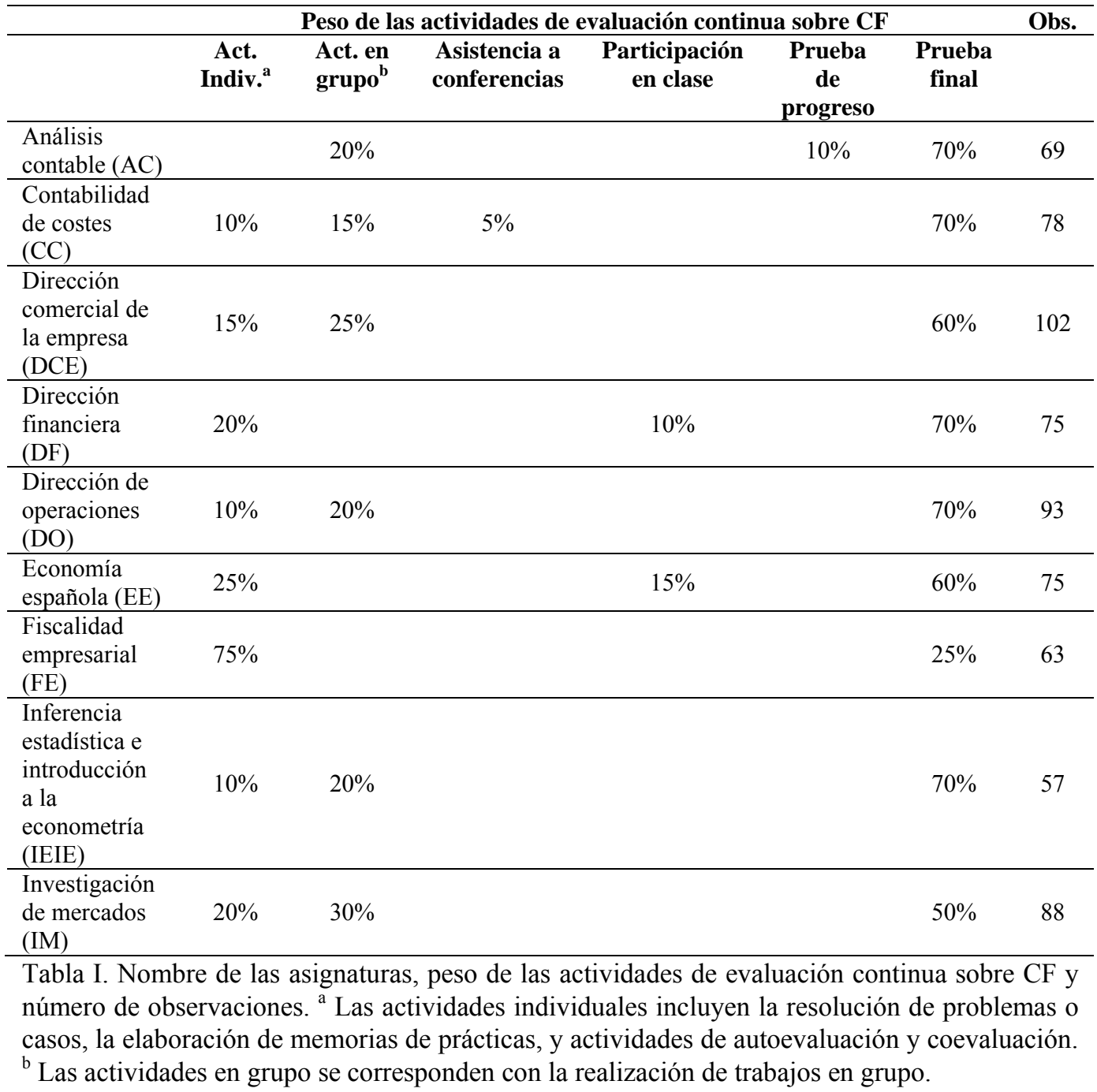

